\title{
Alteration in amyloid $\beta 42$, phosphorylated tau protein, interleukin 6, and acetylcholine during diabetes-accelerated memory dysfunction in diabetic rats: correlation of amyloid $\beta 42$ with changes in glucose metabolism
}

You Zhou' ${ }^{1}$ Ying Zhao ${ }^{1 *}$, Hailong Xie ${ }^{1,2}$, Yan Wang ${ }^{1}$, Lin Liu ${ }^{1}$ and Xinjia Yan ${ }^{1}$

\begin{abstract}
Background: Diabetes accelerates memory dysfunction in a continuous, slowly pathological process. Studies suggest that the time course of certain biomarkers can characterize the pathological course of the disease to provide information for early intervention. Thus, there is an urgent need for validated biomarkers to characterize the cognitive impairment induced by DM. We aimed to detect changes in cerebrospinal fluid biomarkers such as amyloid $\beta 42$, phosphorylated tau protein, interleukin 6 , and acetylcholine in diabetic rats over time, and to analyse the relationship between diabetes and cognitive impairment.

Methods: Rats were injected once intraperitoneally with $1 \%$ of streptozotocin to establish a diabetic model. Index changes were investigated longitudinally and all were measured at the end of the experiment at day 75. A 42 , P-tau, IL-6, and ACh levels in CSF, insulin levels in plasma, and A $\beta 42$ levels in plasma and brain tissue were measured by ELISA.

Results: Compared with control, the diabetic model showed ACh in CSF to be decreased by day 15, continuing lower out to day 75. A 42 changes in brain and blood showed the same trends but exhibited minima at different time points: day 30 in CSF and day 15 in plasma. After the minimum, A 342 in cerebrospinal fluid rose and levelled off lower than in the control group, whereas A $\beta 42$ in plasma rose and went above the controls at day 30 , slowly trending upwards for the remainder of the experiment. P-tau protein in CSF in diabetic rats showed an increasing trend, becoming significantly different from the controls at day 60 and day 75 . A 42 in CSF was strongly negatively correlated with blood glucose at day 15 and was negatively correlated with insulin in serum, particularly at day 45 .

Conclusion: Our longitudinal research model suggest that changes in the measured biomarkers appear before learning and memory impairments do. A 42 and ACh in the diabetes model group clearly changed from day 0 to day 45 , and then P-tau and IL-6 varied significantly from day 45 to day 75 . The reduced ACh levels observed possibly correlated with the factors common to changes in A $\beta 42$, P-tau, and IL-6.
\end{abstract}

Keywords: Diabetes mellitus, Cognitive impairment, Biomarkers, Cerebrospinal fluid

\footnotetext{
*Correspondence: yingzhao16@yahoo.com

${ }^{1}$ College of Pharmacy, Harbin University of Commerce, Harbin,

Heilongjiang 150076, China

Full list of author information is available at the end of the article
} 


\section{Background}

In recent years, the incidence of diabetes mellitus (DM) has been rising at an alarming rate. It has been reported that in China in 2010, the number of DM patients reached 114 million, accounting for $11.6 \%$ of China's adult population and about one-third of total cases worldwide [1]. In research from the Netherlands, an increased risk of dementia was observed in patients suffering from type 2 diabetes [2]. Many studies have demonstrated a sequence of changes in neuropsychological and neurological behaviour in patients with diabetes $[3,4]$, and cognitive impairment with an increased risk of dementia in DM patients has become a serious social problem [5]. Increasing numbers of studies show that DM is an independent risk factor for Alzheimer disease (AD), vascular dementia, and other diseases closely related to $\mathrm{AD}$. The mechanism of vascular dementia involves impairment of the insulin signalling pathway, altered glucose metabolism, metabolic changes such as amyloid $\beta 42$ (A $\beta 42)$ imbalance, phosphorylation of tau protein (P-tau), and release of inflammatory cytokines (e.g. interleukin 6). These changes cause neuronal apoptosis, neuronal damage (particularly in the hippocampus), structural and functional synaptic damage, and neurotransmitter underexpression, collectively leading to cognitive impairment [6].

However, several questions remain concerning the cognitive impairment induced by DM: (1) Is the pathogenesis of the impairment caused by a sequence of phases, e.g. insulin signalling pathway damage, then impaired glucose metabolism, then inflammation? To understand this process, it is urgent to track the changes over time, particularly in insulin, $A \beta 42$, tau protein, inflammatory cytokines, and other biological markers, to investigate the significance for pathogenesis of the intrinsic links among these. (2) What is the time dependence of the mechanism of damage as revealed by biomarkers, and of the resulting learning and memory deficit? The latter question has motivated research into preventative drug intervention in the hope of revealing the best time course of pharmacotherapy. Such research also uncovered the prevalence of blindness in DM.

Considerable evidence shows that diabetes accelerates memory dysfunction in a continuous, slowly pathological process. The incidence is difficult to assess, separate pathological stages are difficult to delineate clearly, and the injury mechanisms predominating at different times during this process continue to require clarification, all of which has hindered research and the formulation of optimal drug intervention strategies. A recent study in the United States suggests that the time course of certain biomarkers can characterize the pathological course of the disease to provide information for early intervention [7]. Thus, there is an urgent need for validated biomarkers to characterize the cognitive impairment induced by DM. It may be possible to interfere with the trajectory of the targeted biological markers in different phases of pathogenesis, and thus to provide the starting point for intervention in the disease process to prevent further damage. Studies have shown that during diabetes-induced cognitive impairment, metabolic and biochemical changes occur in the brain earlier than changes in function or structure. The metabolic changes comprise imbalances between amyloid $\beta(\mathrm{A} \beta)$ production and clearance, abnormal phosphorylation of tau protein, altered IL- 6 levels (also associated with estimated lifetime cognitive decline [8]), lack of neurotransmitters, and structural and functional damage to neurons, ultimately leading to cognitive dysfunction. Both the learning and memory impairment, which influence each other, and the metabolic changes induced by DM causing damage to neurons (as evidenced by structural and functional studies), are progressive. In light of our previous results, we put forward the following hypothesis: 'Changes in brain A $\beta$, P-tau protein, IL-6, and ACh are the first early events in the memory impairment process, and after a delay, which indicates the intervention time window, may cause the cognitive impairment observed in DM'. It is necessary to establish that the brain damage in DM is secondary to ensure that delaying or preventing the earliest pathological and functional changes will be effective. However, continuous dynamic multi-marker locus studies on cognitive impairment in diabetes have not yet been reported. Therefore, this study aimed to chart the time course of the biomarker changes-the initial memory impairment events-during the latency period of DM-induced cognitive impairment. The diabetes model was the streptozotocin (STZ)-injected rat, the biomarkers chosen for study were the CSF levels of A $\beta 42$, P-tau, IL-6, and ACh, and the behavioural assessment method for learning and memory ability was the eight-arm maze test. We also looked at blood chemistry to obtain a better view of the entire pathological process, thereby providing a better understand of the stages of cognitive impairment induced by DM and experimental evidence to guide drug intervention. The study will also provide further evidence bearing on whether DM causes cognitive impairment.

\section{Methods}

\section{Experimental animals}

Male Wistar rats, 8-10 weeks old, weighing $200 \pm 20$ g, were obtained from the Beijing Vital River Laboratory Animal Technology Co., license number SCXK (Beijing) 2012-0001. The animal groups were housed separately in a 12-h alternating light-dark environment, with a temperature of $18-21^{\circ} \mathrm{C}$ and relative humidity of $50 \pm 5 \%$. 
The experimental protocol was approved by the Animal Ethics Committee of the Harbin University of Commerce and was carried out in accordance with the Guidance Suggestions for the Care and Use of Laboratory Animals, issued by the Ministry of Science and Technology of China [9].

\section{Diabetes model}

Before the experiment, the animals were fasted for $12 \mathrm{~h}$ with water freely available. STZ stock solution was freshly prepared by dissolving STZ in citrate-sodium citrate buffer on an ice bath with protection from light, to give a $1 \%$ solution ( $\mathrm{pH} 4.2-4.4$ ). To establish the diabetes model, we administered an intraperitoneal injection of STZ stock solution at a dose of $55 \mathrm{mg} / \mathrm{kg}$ for the model group ( $\mathrm{n}=50$ ), completing the injection within $10 \mathrm{~min}$. We injected citric acid-sodium citrate buffer at the same volume $(5.5 \mathrm{~mL} / \mathrm{kg})$ for the control group $(\mathrm{n}=12)$. Seventytwo hours after injection, blood glucose was determined using a kit (Built Technology Co., Ltd., Nanjing, China), and animals with a fasting plasma glucose $\geq 16.7 \mathrm{mmol} / \mathrm{L}$ [10] were selected for inclusion in the experiment.

\section{Cerebrospinal fluid dynamic acquisition}

CSF was continuously collected via the cisterna magna using a slightly modified version of a published procedure [11]. In a one-time procedure for a given animal, an intravenous infusion needle was slowly inserted to reach the cerebellar cistern, and the other end was connected to a 1-mL syringe, permitting slow collection of cerebrospinal fluid. After animal surgery, the wound was closed and the animals placed singly in cages for 5-7 days for wound healing. Once the diabetes model was established, CSF was collected every 15 days from each rat.

\section{Determination of A $\beta 42$, P-tau, IL-6, and ACh in CSF, and $A \beta 42$ content in brain tissue by ELISA}

The A $\beta 42$, P-tau, IL-6, and ACh contents of CSF were determined using ELISA kits (Bluegene Biotech Co., Shanghai, China). The microplate absorbance was measured at $450 \mathrm{~nm}$. Diabetic rat brain tissues were taken at d75 and the homogenate centrifuged $(15,000 \mathrm{rpm}$, $10 \mathrm{~min}, 4^{\circ} \mathrm{C}$, Sigma $\left.3 \mathrm{~K} 30\right)$. A $\beta 42$ level in the supernatant was measured by ELISA. A kit based on the Bradford method (Nanjing Jiancheng Technology Co., Ltd., Nanjing, China) was used to measure tissue protein content. The A $\beta 42$ content of brain tissue was expressed as picograms per milligram of total protein.

\section{Learning and memory ability of rats determined by the eight-arm maze test}

After the diabetic rat model had been established for 60 days, learning and memory were tested by the eight-arm radial maze using a video behavioural analysis system provided by the Huaibei Zhenghua Biological Equipment Co., Ltd. (ZH-3000 type, Anhui, China). After completion of adaptive training, diet was restricted to $85 \%$ of ad libitum daily consumption by weight. The rats were placed in the central area of a maze with an opaque cover, and the arm doors were opened. Behaviour was recorded for $10 \mathrm{~min}$. Rats entered each arm from the central area searching for food, and entering an arm was considered to have occurred when $70 \%$ of the body was inside the door to the arm. If the food had not been finished after $10 \mathrm{~min}$, the test was terminated. Rats were tested once daily for 5 days. Working memory errors, reference memory errors, and total foraging time were recorded.

\section{Plasma A $\beta 42$ and indicators of glucose metabolism}

Before plasma sampling, rats were fasted for $12 \mathrm{~h}$ with water available. Blood $(0.5 \mathrm{~mL})$ was then collected from the retinal venous plexus and centrifuged (5,000 rpm, $10 \mathrm{~min}$ ) to separate the plasma. Plasma glucose was determined by the glucose oxidase method using a kit (Nanjing Jiancheng Technology Co., Ltd., Nanjing, China). Plasma insulin and A $\beta 42$ levels were measured by ELISA using a kit (Bluegene Biotech Co., Shanghai, China).

\section{Statistical analysis}

SPSS 21.0 for Windows was applied for statistical data processing, and results were expressed as means \pm standard deviations. Groups were compared using the independent-samples $t$ test. The Pearson correlation test was used for evaluation of relationships between the various biomarkers (ACh, A $\beta 42$, P-tau, IL-6, insulin, glucose, and other indicators). $P<0.05$ was adopted as the significance level for both $t$ - and Pearson tests.

\section{Results}

Weight and general characteristics observed in diabetic rats

Compared with the control group, rats in the experimental diabetic model group showed apathy; curled, loose stools; wet litter; a heavy smell of urine; and other phenomena. Seventy-two hours after intraperitoneal injection of STZ, the rats exhibited overeating, over drinking, and polyuria, a syndrome consistent with the clinical symptoms of diabetes. As in human diabetes, weight loss was observed (Fig. 1).

Fasting plasma glucose and insulin levels in diabetic rats Our study found that 43 of 50 rats in experimental diabetic model group had elevated blood glucose levels, defined as higher than $16.7 \mathrm{mmol} / \mathrm{L}$, at $72 \mathrm{~h}$ (0 day); 


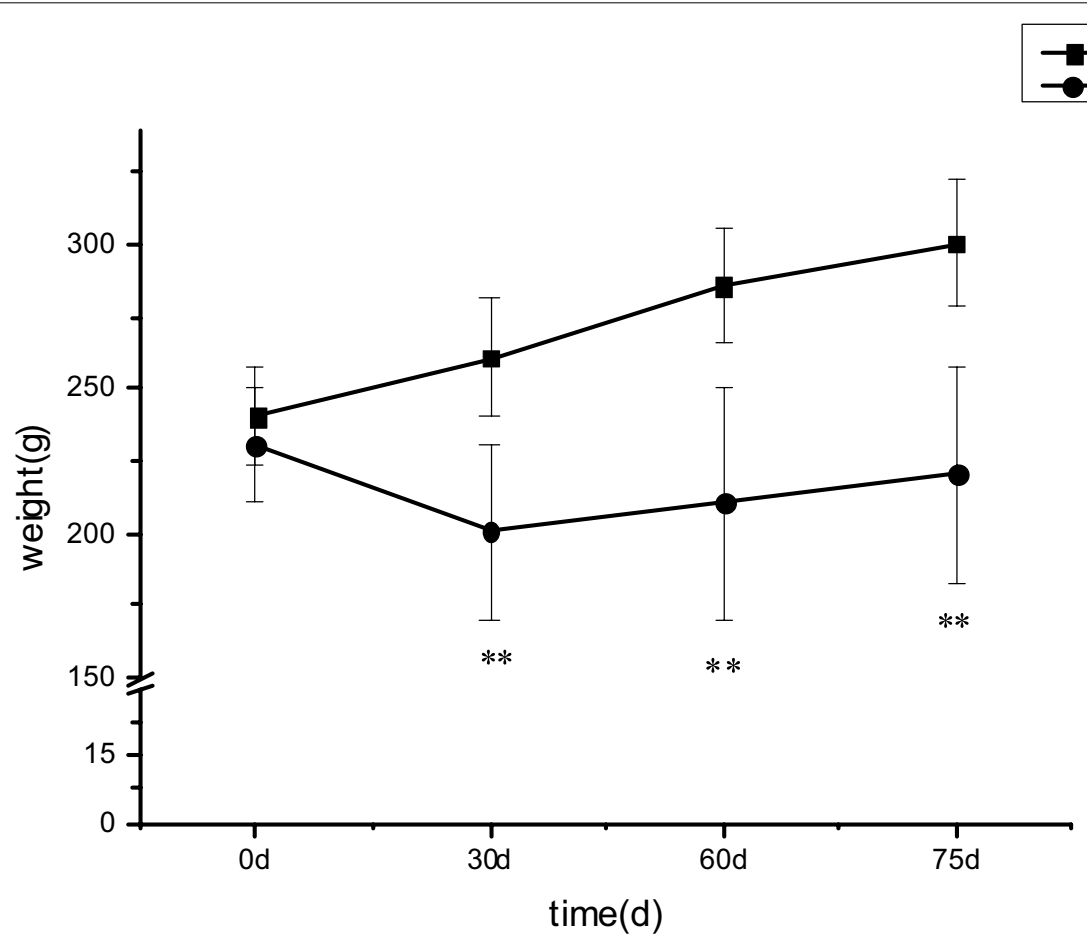

Fig. 1 Dynamic changes in rat body weight. In this figure, model rats (streptozotocin-treated group, $n=15$ ) are compared with control rats (control group, $\mathrm{n}=12$ ). Means \pm standard deviations are shown. For intergroup comparisons: ${ }^{*} P<0.05,{ }^{* *} P<0.01$.

these rats were incorporated into the experiment. In the remaining animals, four died and three showed blood glucose levels not significantly changed. Compared with the control group, glucose was significantly increased in the experimental diabetic model group at day 30, day 60 , and day $75(P<0.01)$. Insulin in plasma was elevated in diabetic rats at day 15 , day 30 , day 45 , and day 60 $(P<0.05$ or 0.01$)$; However, at day 75 , plasma insulin in diabetic rats was reduced compared with that in the same group at day $45(P<0.05)$ (Fig. 2$)$.

\section{Dynamic changes in Aß42, P-tau, IL-6, and ACh levels in CSF of diabetic rats}

During the experiment, CSF was collected six times from diabetic rats over the period day 0 to day 75 . Compared with the control group, A $\beta 42$ was lower in the CSF of diabetic rats at day 15 and at day $30(P<0.05$ or 0.01 , respectively); it was also lower at day 30 compared with previous levels in the same group at day $15(P<0.01)$. Model levels were also higher at day 45 , day 60 , and day 75 than in the same group at day $30(P<0.01)$ (Fig. 3a). Compared with the control group, P-tau levels in the CSF of the experimental diabetic model group were significantly increased at day 60 and day $75(P<0.01)$ (Fig. 3b). IL-6 was also increased in the CSF of the model group at day 60 and day $75(P<0.01)$ (Fig. 3c). Compared with the control group, ACh in the CSF of the diabetic rat model was severely lowered at day $15(P<0.01)$, continuing so to day 75 (Fig. 3d).

\section{Determination of $A \beta 42$ content in the blood of diabetic}

\section{rats}

Compared with the control group, $\mathrm{A} \beta 42$ in the blood of the diabetic rats was significantly lower at day 15 $(P<0.05)$ and significantly higher at day 45 and day 75 $(P<0.05$ and 0.01 , respectively). The A $\beta 42$ levels in the blood of diabetic rats at day 30 , day 45 , and day 75 were significantly increased compared with those in the same group at day $15(P<0.01)$. The A $\beta 42$ levels in the blood of diabetic rats at day 75 were also significantly increased compared with those at day $30(P<0.05)($ Fig. $4 a)$.

\section{Determination of $A \beta 42$ content in the brain tissue of diabetic rats}

Compared with the control group, $A \beta 42$ content in the brain tissue of diabetic rats was found to be increased by day $75(178.99 \pm 44.47 \mathrm{pg} / \mathrm{mg}$ prot; $P<0.05)$ (Fig. $4 \mathrm{~b})$.

\section{Correlation analysis of various biomarkers in diabetic rats}

Pearson correlation analysis showed that changes in $\mathrm{ACh}$ levels showed a high negative correlation with $\mathrm{A} \beta 42$, P-tau, and IL-6 in the CSF of diabetic rats, $r=-0.916$ 


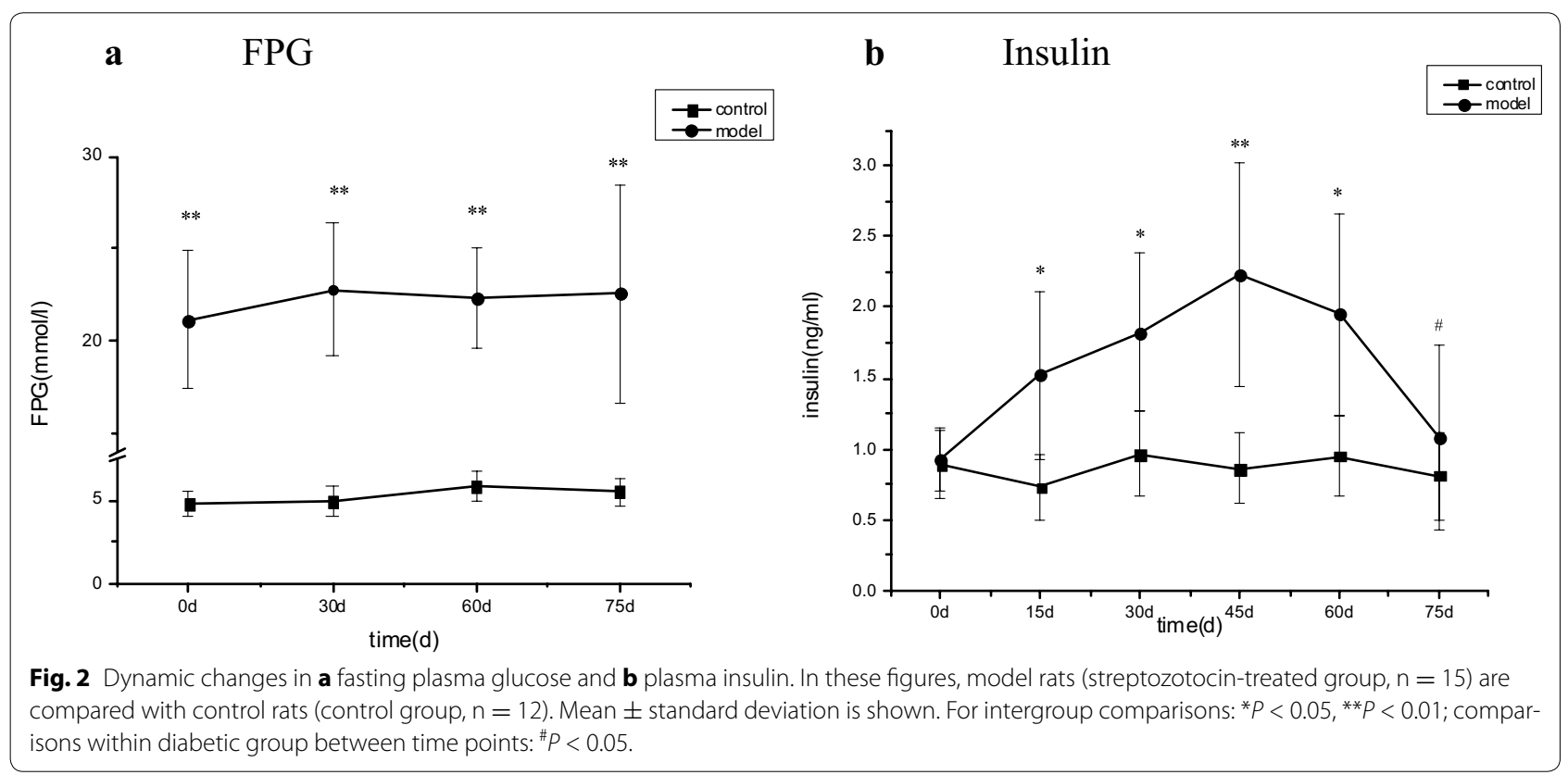

$(P<0.05)$. In addition, at day 0 of the diabetes model, the indicators were not correlated; at day 15 of the model, A 342 in CSF showed a strong negative correlation with blood glucose, $r=-0.865(P<0.05)$. At day 45 of the model, A $\beta 42$ in CSF was strongly negatively correlated with insulin, $r=-0.760(P<0.05)$ and insulin resistance index, $r=-0.946(P<0.05)$. At day $75, \mathrm{~A} \beta 42$ in the blood and brain in the experimental diabetic model group showed low correlation (Tables 1, 2).

\section{Determination of learning and memory ability by the eight-arm maze test in diabetic rats}

Foraging time was significantly longer in diabetic rats compared with control $(P<0.05)$. The number of reference memory errors was also increased significantly in diabetic rats compared with control $(P<0.05)$, suggesting that learning and memory ability in the diabetic rats were damaged by day 71 (Table 3 ).

\section{Discussion}

With the rapid recent increase in the incidence of diabetes, cognitive impairment induced by diabetes has attracted wide attention [1]. Since 2006, when Mijnhout [12] proposed a 'diabetes-related cognitive impairment', several studies have shown that diabetic patients and animal models exhibit cognitive dysfunction. Takeda's research deepened understanding of the mechanistic links between the two types of disease [13]. Recent studies have shown unimpaired cognition in the AD process at the time when $A \beta$ deposition occurs, and only after a delay are neurological dysfunction and degeneration seen
[14]. We therefore have reason to believe that the brain levels of $A \beta$, P-tau protein, IL-6, and ACh mark early events in memory impairment.

From previous literature, it appears that there is no standard cognitive dysfunction induced by diabetic animal models; usually a DM model was established using a drug, and with extension of modelling time, cognitive function was observed to decline. The present study uses a single intraperitoneal injection of STZ to establish the model and tests were conducted over 75 days. During the experiment, the blood glucose in the experimental diabetic model group was stable at above $16.7 \mathrm{mmol} / \mathrm{L}$, showing good model stability.

In this study, the various biomarker changes were followed by collecting CSF from the rats, methods for which are broadly divided into two categories: the lateral ventricle puncture, involving drilling a hole in the animal's skull and inserting a drainage tube for collecting cerebrospinal fluid [15], and the extraction of CSF via a needle puncture into the cisterna magna [11]. Our experiment used a method based on a previous publication describing a one-time technique for collection through an intravenous infusion needle. We slightly modified this to a technique for multi-time-point collection from a single animal. This enabled us to collect CSF from diabetic rats longitudinally over $0-75$ days. This allowed us to avoid the need to sacrifice animals at each time point, thereby reducing the standard deviation of the data. As a result, dynamic biomarker changes were observed when DM caused major cognitive damage. A $\beta 42$ and ACh in brain were the first to change significantly; P-tau and 


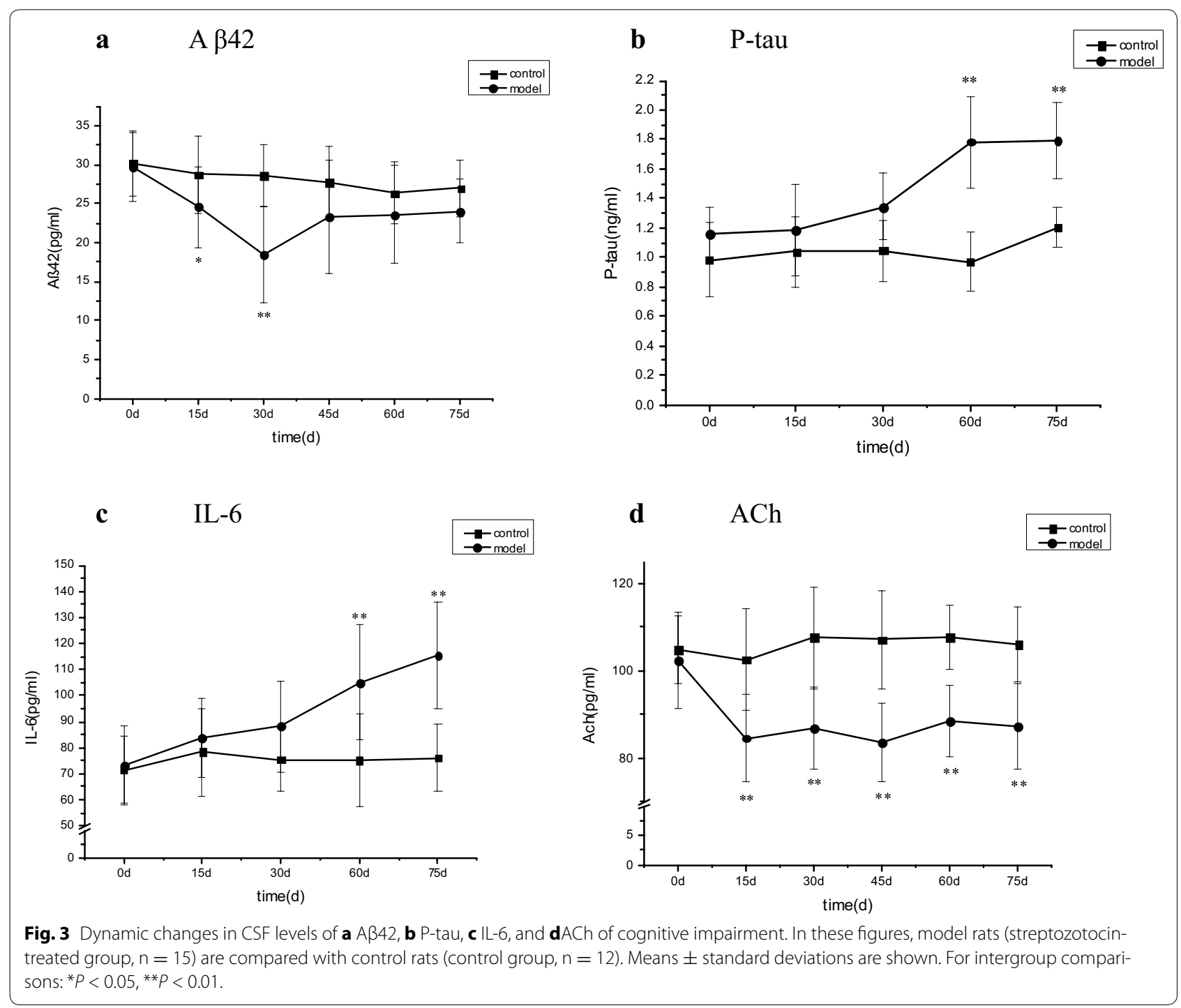

IL-6 protein concentrations changed later and in a more gradual, progressive manner. Currently, there are reports of longitudinal clinical studies of biomarkers of cognitive impairment; however, previous studies tended to explore more than a single pathology, rarely also discussing two or more dynamic changes in biomarkers or the long-term pathological evolution (e.g., over 2 months). In the present study, we collected cerebrospinal fluid from the cisterna magna five or six times per animal at several time points. The amount of CSF that could be collected per animal per time point was limiting in this experiment because of practical issues with diabetic rats; therefore, not all the indicators were measured at some time points. However, from related references and pilot experiments, we knew that diabetic rats have a cognitive impairment detectable from day 60 to day 70, and therefore, we knew the appropriate time to terminate the experiment and collect larger, adequate samples from all the animals. Before and after data were therefore available for all biomarkers. Because of the abovementioned limitation, our laboratory intends to perform studies using non-invasive CSF collection methods, seeking methods by which it will be practical to collect cerebrospinal fluid at shorter time intervals, allowing better data to be obtained in future experiments.

The prevailing view is that diabetes is closely related to brain aging and dementia. Dementia is thought to result from an imbalance between $A \beta$ production and clearance, leading to loss of neurotransmitter expression and damaged synaptic function [16, 17]. This study found at day 15 a strong negative correlation of CSF levels of A $\beta 42$ with blood glucose. Accordingly, we have reason to believe that, with the establishment of the diabetes model at day 0 -day 15 , elevated blood glucose can cause 

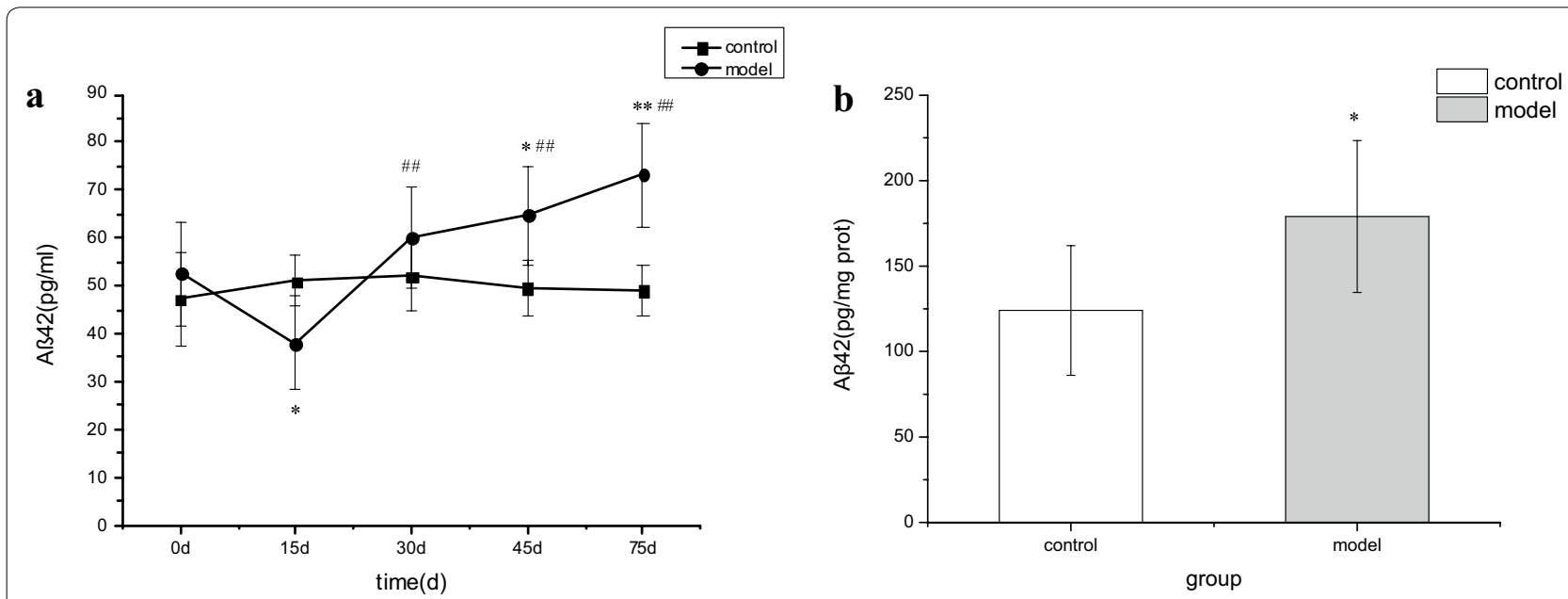

Fig. 4 a Dynamic changes of $A \beta 42$ in plasma of diabetic rats. In these figures, model rats (streptozotocin-treated group, $n=15$ ) are compared with control rats (control group, $\mathrm{n}=12$ ). Mean \pm standard deviation is shown. For intergroup comparisons: ${ }^{*} P<0.05$, ${ }^{* *} P<0.01 ;$ comparisons within diabetic group between time points: ${ }^{\#} P<0.01$. b Brain tissue $A \beta 42$ concentrations at day 75 ( $\left.n=10-12\right)$. For intergroup comparisons: ${ }^{*} P<0.05$.

a severe precipitation phenomenon in $\mathrm{A} \beta 42$ in vivo, $\mathrm{A} \beta 42$ in CSF showing a decline in the early stages of diabetes. It is known that $A \beta 42$ levels in CSF inversely reflect the brain content of the fibrous, insoluble form of $A \beta$, which is the core component of senile plaques and is important in their formation. Its precipitation occurs early in AD $[18,19]$. In our model, the interval 15-30 days, when changes in blood glucose had stabilized, was instructive. Changes in A $\beta 42$ levels in CSF bottomed later than did those in the blood, and we therefore speculate that at this time $A \beta$ deposition may begin to appear in the brain. At day 30, A $\beta 42$ in CSF hits 'rock bottom' due to the double impact of deposition and glycosylation.

It has been reported that hyperinsulinaemia in patients with chronic insulin resistance carries a significantly increased risk of cognitive dysfunction or dementia [20]. Watson et al. found that in healthy older people who inject insulin, both insulin level and $A \beta 42$ level could be increased in CSF [21]. In the present study, with synchronous dynamic monitoring of plasma insulin levels in diabetic rats, we found that when the model plasma insulin levels were increased vs. controls (day 30-day 45), a peripheral insulin resistance phenomenon being dominant, the plasma $A \beta 42$ level was also significantly increased vs. controls, presumably due to A $\beta 42$ degradation and insulin degradation competing for the same substrate-insulin-degrading enzyme [22, 23]. In the diabetes model, day 45-day 75 is a period of falling plasma insulin levels, which may be associated with a negative feedback mechanism that reduces insulin levels by upregulating expression of insulin-degrading enzyme, accelerating A $\beta 42$ degradation as well. However, the enzyme was again inhibited, leading to elevated blood levels of
A $\beta 42$. At the same time, correlation analysis at diabetic model day 45 showed A $\beta 42$ in CSF to be negatively correlated with plasma insulin and insulin resistance index. By observing changes over time, it can be seen that day 45 is the watershed of insulin level change, marking the beginning of a decrease in insulin levels. At this time point, cerebrospinal fluid $A \beta 42$ levels began to rise, the opposite trend, showing a negative correlation.

It is known that P-tau concentration reflects the level of neurofibrillary tangles in the brain. This study found that changes in P-tau in the CSF of rats in our diabetic model group occurred later than the changes in A $\beta 42$; a significant increase in P-tau in CSF was observed only at day 60 and day 75. Studies have shown that after a fall in peripheral insulin levels, the insulin signalling pathway is down-regulated, resulting in the downstream phosphatidylinositol 3-kinase activity being decreased while the PI-3K activity is decreased, which will cause downstream tau protein phosphorylation [24]. The main kinase, glycogen synthase kinase- $3 \beta$, passes from a non-activated to an activated state. Thus, there will be hyperphosphorylation of tau protein leading to AD-like changes [25]. Therefore, we speculate that abnormal PI-3K pathway activation of glycogen synthase kinase-3 $\beta$ in an era of declining insulin caused tau protein phosphorylation to be increased in our model.

Analysis shows that the trajectory changes were different over time. In diabetic rats at each time point, fluctuations in A $\beta 42$ and P-tau protein concentration in CSF appeared complementary, namely 'shift' changes over time. P-tau protein was on a gradually increasing trend when $A \beta 42$ was decreased relative to controls, which is consistent with clinical reports [26]. The experiment 


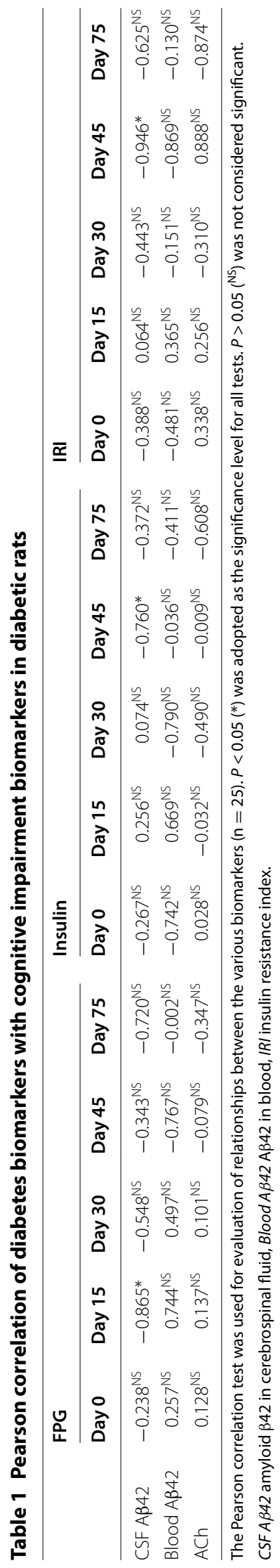


Table 2 Correlation analysis of $A \beta 42$ in blood and cerebrospinal fluid in diabetic rats

\begin{tabular}{lcc}
\hline Time point & Pearson correlation & $P$ value \\
\hline Day 0 & $0.327^{\mathrm{NS}}$ & 0.295 \\
Day 15 & $-0.647^{\mathrm{NS}}$ & 0.174 \\
Day 30 & $-0.623^{\mathrm{NS}}$ & 0.283 \\
Day 45 & $0.604^{\mathrm{NS}}$ & 0.140 \\
Day 75 & $-0.693^{\mathrm{NS}}$ & 0.256
\end{tabular}

The Pearson correlation test was used. $P<0.05\left({ }^{*}\right)$ was adopted as the significance level for all tests. $P>0.05\left({ }^{\mathrm{NS}}\right)$ was not considered significant. Results are shown for day 0 to day 75 in diabetic model rats $(n=15)$.

Table 3 Learning and memory capacity in diabetic rats at day 71-eight-arm maze test

\begin{tabular}{llll}
\hline Group & $\mathbf{n}$ & Foraging time (s) & $\begin{array}{l}\text { Number of reference } \\
\text { errors }\end{array}$ \\
\hline Control & 10 & $366.5 \pm 64.8$ & $2.78 \pm 0.33$ \\
Model & 15 & $505.5 \pm 53.3^{*}$ & $5.40 \pm 0.85^{* *}$ \\
\hline
\end{tabular}

A rat diabetes model (streptozotocin-treated group, $n=15$ ) is compared with control $(n=10)$. Means \pm standard deviations are shown. For intergroup comparisons: ${ }^{*} P<0.05,{ }^{* *} P<0.01$.

found that in individual diabetic animals, lower $A \beta 42$ in CSF at early times (day 15-day 30 time-points) appears to reduce the phenomenon of phosphorylated tau protein. The experiment also found that IL- 6 expression at model day 60 -day 75 was increased $(P<0.01)$, plasma A $\beta 42$ at model day 15 was decreased $(P<0.01)$, and $\mathrm{A} \beta$ expression in rat brain tissue at model day 75 was increased $(P<0.05)$. These results are consistent with previous reports [27].

Currently, intraperitoneal injection of STZ to induce learning and memory dysfunction in rodent models has academic recognition [28]. In agreement with this, the present study found that in the diabetes model at day 71 , foraging time in the eight-arm maze was significantly longer than in control rats. Our results suggest that changes in the measured biomarkers seem to appear earlier than learning and memory impairments since according to the work of others cognitive deficits do not appear earlier than 70 days after diabetes induction [29].

The above the trajectory analysis of biomarker change shows that reduced levels of A $\beta 42$ in CSF is an early event, and that P-tau protein and IL- 6 changes are later events. The above metabolic changes together determine the cognitive dysfunction in diabetic rats, and our study supports the hypothesis previously proposed, namely that changes in brain A $\beta 42$, P-tau protein, IL-6, and ACh expression are the early events in diabetic memory impairment, and the latency period of the latter may provide an intervention time window for preventing cognitive impairment in DM. Moreover, deposition of brain $\mathrm{A} \beta 42$ predicts a cognitive-impairment pathological process, and leads to synaptic dysfunction, culminating in cell loss and brain atrophy. Cognitive impairment induced by diabetes is characterized by a diversity of mechanisms, which dictates multi-target intervention strategies. At the same time, changes in the trajectory of different biomarkers at different stages of cognitive impairment due to the different mechanisms of injury determine drug selection. Another purpose of this experiment was to study drug intervention strategies. Correlation analysis showed that $\mathrm{ACh}, \mathrm{A} \beta 42$, P-tau, and IL-6 levels in the CSF of diabetic rats were not linearly related $(P>0.05)$, while further data analysis showed that ACh change and A $\beta 42$, P-tau, and IL-6 were highly negatively correlated, suggesting that changes in $\mathrm{ACh}$ in diabetic rats were determined by the common factors in $A \beta 42$, P-tau, and IL-6 variation. In the diabetes model at day 0-day 30, P-tau and IL-6 are not a significant source of variation, $A \beta 42$ and $A C h$ being the main factors changing. A $\beta 42$ content in CSF was reduced, reaching a minimum at day 30. In light of the above analysis, the results suggest that in terms of choice of drug intervention strategies, blood glucose should be controlled to rectify the early $A \beta 42$ protein deposition, etc., due to the double impact of glycosylation and insulin elevation, thus preventing possible cognitive damage. This study also found increases in P-tau and IL- 6 protein in CSF in the diabetic model at day 60 -day 75 , but by this time $A \beta 42$ changes had stabilized. At this stage, it is believed that the main factors causing cognitive impairment effects are decreased ACh content and increased P-tau protein and IL-6 in CSF. Based on the above considerations, targeted intervention therapy with drugs to ameliorate insulin resistance and combat inflammation should be considered the best choice for this latter stage.

This study has some limitations, such as the lack of effective mathematical methods for studying the quantitative characteristics of the biomarkers. In future, we intend to use mathematical methods to reveal the quantitative relationships among the various markers and the dependence of each marker on time. Moreover, the study did not use a model appropriate to proving the effectiveness of specific drug intervention strategies. In subsequent experiments, these problems will be further addressed.

\section{Conclusions}

We found that the trajectories of A $\beta 42, \mathrm{P}-\mathrm{tau}, \mathrm{IL}-6$, and $\mathrm{ACh}$ levels in CSF were different in dissimilar stages of diabetes-accelerated memory dysfunction in rats. Results from our longitudinal research model suggest that changes in the measured biomarkers appear before 
learning and memory impairments do. In the diabetes model group, $\mathrm{A} \beta 42$ and $\mathrm{ACh}$ changed obviously from day 0 to day 45, after which P-tau and IL- 6 varied significantly from day 45 to day 75 . In our diabetes model, A $\beta 42$ in CSF may be correlated with blood glucose in rats from day 0 to day 45 , after which it correlates strongly with insulin. The reduced ACh levels observed possibly correlated with the factors common to changes in A $\beta 42$, P-tau, and IL-6.

\section{Abbreviations}

A 342: amyloid $\beta 42$; P-tau: phosphorylated tau protein; IL-6: interleukin 6; ACh: acetylcholine; AD: Alzheimer disease; DM: diabetes mellitus; STZ: streptozotocin; CSF: cerebrospinal fluid.

\section{Authors' contributions}

All authors listed have contributed to the work. YZ and HX participated in design of the experimental protocol, data collection, statistical analysis, and writing of the manuscript. LL, YW, and XY carried out the behavioural tests and sample collection. $Y Z$ is the corresponding author. All authors read and approved the final manuscript.

\section{Author details}

${ }^{1}$ College of Pharmacy, Harbin University of Commerce, Harbin, Heilongjiang 150076, China. ${ }^{2}$ College of Pharmacy, Heilongjiang University of Chinese Medicine, Harbin, Heilongjiang 150040, China.

\section{Acknowledgements}

This study was funded by the following agencies, to which we extend our sincere appreciation.

National Natural Science Foundation of China (Grant No. 81373548).

The 2013 Heilongjiang Province Natural Science Fund Project (Grant No. H201382).

The 2012 Joint Specialized Research Fund for the Doctoral Program of Higher Education (Grant No. 20122332110004).

\section{Compliance with ethical guidelines}

\section{Competing interests}

The authors declare that they have no competing interests.

Received: 25 January 2015 Accepted: 14 July 2015

Published online: 14 August 2015

\section{References}

1. Xu Y, Wang L, He J et al (2013) Prevalence and control of diabetes in Chinese adults. JAMA 310:948-959

2. Ott A, Stolk RP, Van Harskamp F et al (1999) Diabetes mellitus and the risk of dementia. The Rotterdam Study. Neurology. 53:1937-1942

3. Matsuzawa T, Takata T, Yokono K et al (2012) A warning index used in prescreening for Alzheimer's disease, based on self-reported cognitive deficits and vascular risk factors for dementia in elderly patients with type 2 diabetes. J Alzheimers Dis, Int, pp 1-8

4. Hai Yan YU, Qun Song WANG, Ping LI et al (2011) The placebo controlled study of type 2 diabetes patients with mild cognitive impairment. Chin J Gerontol 31:4087-4089

5. Shuba N (2012) Assessment of the cognitive status in diabetes mellitus. J Clin Diagn Res 6:1658-1662

6. Kimura R, Tomiyasu H, Takeuchi T et al (2008) Prevalence of Alzheimer's disease with diabetes in the Japanese population. Psychogeriatrics. 8:73-78
7. Rosenberg RN (2011) Treat Alzheimer disease before it is symptomatic. Arch Neurol 68:1237-1238

8. Marioni RE, Strachan MW, Reynolds RM, Lowe GD, Mitchell RJ, Fowkes FG et al (2010) Association between raised inflammatory markers and cognitive decline in elderly people with type 2 diabetes. Diabetes 59:710-713

9. The Ministry of Science and Technology of the People's Republic of China (2009) Guidance suggestions for the care and use of laboratory animals

10. Wei W (2010) Experimental methodology of pharmacology (M), 4th edn. People's medical publishing house, Beijing, pp 1364-1368

11. Huang YL, Säljö A, Suneson A et al (1995) A new approach for multiple sampling of cisternal cerebrospinal fluid in rodents with minimal trauma and inflammation. J Neurosci Methods 63:13-22

12. Mijnhout GS, Scheltens P, Diamant M et al (2006) Diabetic encephalopathy: a concept in need of a definition. Diabetologia 49:1447-1448

13. Takeda S, Sato N, Uchio-Yamada K et al (2010) Diabetes-accelerated memory dysfunction via cerebrovascular inflammation and $A \beta$ deposition in an Alzheimer mouse model with diabetes. Proc Natl Acad Sci 107:7036-7041

14. Lo RY, Hubbard AE, Shaw LM et al (2011) Longitudinal change of biomarkers in cognitive decline. Arch Neurol 68:1257-1266

15. Ehlert A, Tiemann B, Elsner J et al (2010) Long-term subarachnoid catheter placement in the middle cranial fossa of the rat. Lab Anim. 39:352-359

16. Sheng SL (2001) Diabetic encephalopathy and Alzheimer's disease. Chin J Endocrinol Metab. 17:58-59

17. Fazeli SA (2010) Neuroprotection in diabetic encephalopathy. Neurodegenerative Diseases. 6:213-218

18. Zheng MG, Ruan ZG, Jing LIU, LI GY (2011) Metabolism mechanism of Beta amyloid protein. Chin J Neuroanat 27:344-348

19. Fernandez-Gamba A, Leal MC, Morelli L et al (2009) Insulin-degrading enzyme: structure-function relationship and its possible roles in health and disease. Curr Pharm Des 15:3644-3655

20. Peila R, Rodriguez BL, White LR et al (2004) Fasting insulin and incident dementia in an elderly population of Japanese-American men. Neurology. 63:228-233

21. Watson GS, Craft S (2003) The role of insulin resistance in the pathogenesis of Alzheimer's disease. CNS drugs. 17:27-45

22. Carrasquillo MM, Belbin O, Zou F, Allen M, Ertekin-Taner N, Ansari M et al (2010) Concordant association of insulin degrading enzyme gene (IDE) variants with IDE mRNA, Abeta, and Alzheimer's disease. PLoS One 19:e8764

23. Farris W, Mansourian S, Chang Y et al (2003) Insulin-degrading enzyme regulates the levels of insulin, amyloid beta-protein, and the beta-amyloid precursor protein intracellular domain in vivo. Proc Natl Acad Sci USA 100:4162-4167

24. Nemoto T, Yanagita T, Kanai T, Wada A (2009) Drug development targeting the glycogen synthase kinase-3beta-mediated signal transduction pathway: the role of GSK-3beta in the maintenance of steady-state levels of insulin receptor signalling molecules and $\mathrm{Na}(\mathrm{v}) 1.7$ sodium channel in adrenal chromaffin cells. Pharmacol Sci 109:157-161

25. Ciaraldi TP, Carter L, Mudaliar S, Henry RR (2010) GSK-3beta and control of glucose metabolism and insulin action in human skeletal muscle. Mo Cell Endocrinol 315:153-158

26. Liu F, Shi J, Tanimukai H et al (2009) Reduced O-GlcNAcylation links lower brain glucose metabolism and tau pathology in Alzheimer's disease. Brain. 132:1820-1832

27. Zhiyou CAl, Yong YAN, Jun ZHANG et al (2009) Cognitive function and expression of GFAP, IL-1B, TNF-A and AB in hippocampal tissues of diabetic rats. J Shanghai Jiaotong University (Medical Science). 129:16-20

28. Mehmet T, Giyasettin B (2006) Effect of melatonin and vitamin E on diabetes induced learning and memory impairment in rats. Eur J Pharmacol 53:106-110

29. Kuhad A, Chopra K (2007) Curcumin attenuates diabetic encephalopathy in rats: behavioral and biochemical evidences. Eur J Pharmacol 576:34-42 